\title{
The Reactivity of Hydroxyl Groups at Different Tactic Sequences on Poly(vinyl alcohol) in the Addition Reaction with Vinyl Sulfoxides and Vinyl Sulfones
}

\author{
Kiyokazu ImaI, Tomoo Shiomi, Yasuyuki TezuKa, \\ and Takashi TsukaHARA \\ Department of Material Science and Technology, Nagaoka University of Technology, \\ Kamitomioka, Nagaoka, Niigata 940-21, Japan
}

(Received March 7, 1991)

\begin{abstract}
The reactivity of hydroxyl groups in different tactic sequences on poly(vinyl alcohol) was studied in the Michael type addition reaction with vinyl sulfoxides and vinyl sulfones. By the ${ }^{13} \mathrm{C}$ NMR anaysis on the methine carbon signal in unreacted vinyl alcohol units for the obtained sulfinylethyl and sulfonylethyl poly(vinyl alcohol)s, it was concluded that the reactivity order of hydroxyl groups in different tractic sequences was isotactic $>$ heterotactic $>$ syndiotactic.
\end{abstract}

KEY WORDS Reactivity / Tacticity / Poly(vinyl alcohol) / Vinyl Sulfoxide /

Vinyl Sulfone / Michael Addition Reaction /

We have recently described new membrane materials for the selective accumulation and the separation of pollutant sulfur dioxide based on sulfoxide- and sulfone-modified polymers produced through a Michael-type addition reaction of hydroxyl groups in poly(vinyl alcohol), ${ }^{1-3}$ cellulose ${ }^{4}$ and ethylene/vinyl alcohol copolymer ${ }^{5,6}$ with a variety of vinyl sulfoxides and vinyl sulfones.

The control on not only the total content of sulfoxide or sulfone groups but also the distribution of functional groups along the polymer chains is considered to be of a significant importance to optimize the permselective property which retaining the sufficient mechanical strength of the product. Since the reactivity of substituent groups along the polymer chain can be influenced by the tactic sequence, we have examined in the present study on the reactivity of hydroxyl groups at the different tactic sequences on poly(vinyl alcohol) in the addition reaction with vinyl sulfoxides and vinyl sulfones.

The reactivity of substituent groups in different tactic sequences has been so far examined for poly(vinyl chloride) in the substitution ${ }^{7-9}$ or the abstraction ${ }^{10}$ reactions, and also for poly(vinyl chloroformate), ${ }^{11}$ where the substituent at the isotactic sequence exhibited higher reactivity than those at the syndiotactic counterpart. It was also shown in the modification reaction of poly(vinyl alcohol) that the acetalization ${ }^{12-15}$ and the ketalization ${ }^{16}$ took place preferentially at the isotactic sequence in which the energitically favorable equatorial-equatorial conformation can be maintained at the produced six-membered 1,3-dioxane ring skeleton.

\section{EXPERIMENTAL}

\section{Reagents}

A series of vinyl sulfoxides and sulfones was synthesized by the method described in the preceding papers. ${ }^{1,2}$ Poly(vinyl alcohol) was obtained from Kuraray Co. (PVA $117 \mathrm{H}$, $\mathrm{DP}=1700$, the degree of saponification: $>99.8 \%$ ) and used after the Soxhlet extraction 


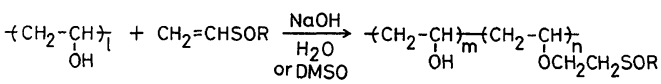

$$
\begin{aligned}
& \mathrm{R}=\mathrm{CH}_{3}, \mathrm{C}_{2} \mathrm{H}_{5}, \mathrm{t}-\mathrm{C}_{4} \mathrm{H}_{9}, \mathrm{C}_{6} \mathrm{H}_{5}
\end{aligned}
$$

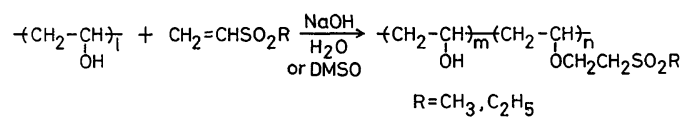

Scheme 1.

with methanol. Dimethylsulfoxide (DMSO) was dried over molecular sieves $4 \mathrm{~A}$. Other reagents were used after conventional purification procedures.

\section{Reaction of Poly(vinyl alcohol) with Vinyl} Sulfoxides and with Vinyl Sulfones

The addition reaction of vinyl sulfoxides and of vinyl sulfones with poly(vinyl alcohol) was performed either in aqueous solution or in DMSO with the addition of sodium hydroxide as a catalyst. ${ }^{1,2}$ The reaction products with vinyl sulfoxides were isolated by the precipitation into either directly acetone-methanol $(8: 2)$ mixture (for the samples of less than $50 \%$ substitution) or acetone after the dialysis (for the samples of more than $50 \%$ substitution). The reaction products with vinyl sulfones were isolated by the precipitation into methanol. The products were then subjected to the repeated precipitation and finally vacuum-dried at $45^{\circ} \mathrm{C}$ for 2 days. The degree of substitution in the products was determined by comparing the ${ }^{1} \mathrm{H}$ NMR signal intensity of substituent groups with main chain groups. ${ }^{1,2}$

\section{NMR Measurements}

${ }^{1} \mathrm{H}$ and ${ }^{13} \mathrm{C}$ NMR measurements were carried out at $270 \mathrm{MHz}$ and at $67.8 \mathrm{MHz}$, respectively, with a JEOL JNM GX 270 apparatus by using a $5 \mathrm{~mm} \phi \mathrm{C}-\mathrm{H}$ dual probe in $\mathrm{D}_{2} \mathrm{O}$ at $40^{\circ} \mathrm{C}$ and the dimethyl sulfoxide- $d_{6}$ (DMSO- $d_{6}$ ) at $60^{\circ} \mathrm{C}$. Chemical shift values were calibrated either from 3-(trimethylsilyl)propionic acid sodium salt- $d_{4}\left(0 \mathrm{ppm}\right.$ in $\left.\mathrm{D}_{2} \mathrm{O}\right)$ or DMSO signal (2.50 ppm for ${ }^{1} \mathrm{H}$ and $43.5 \mathrm{ppm}$

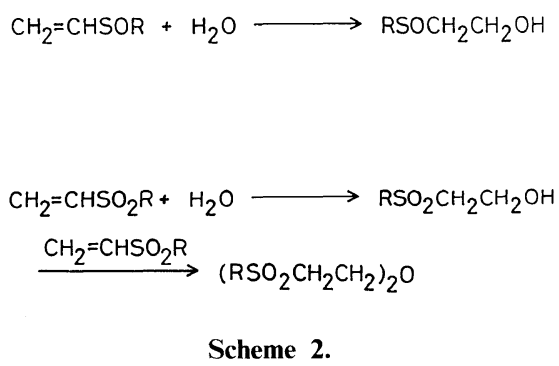

Scheme 2.

for ${ }^{13} \mathrm{C}$ ) as internal standards.

\section{RESULTS AND DISCUSSION}

The addition reaction of vinyl sulfoxides and of vinyl sulfones with poly(vinyl alcohol) was found to proceed both in aqueous and in DMSO solution by adding sodium hydroxide as a catalyst. Nevertheless, the concurrent side reaction was observed between vinyl sulfoxides or sulfones and water present in the reaction mixture either as a solvent or as a medium to solubilize the catalyst in DMSO. Thus the products of high degrees of sulfinylation or sulfonylation were obtained when the reaction was carried out in DMSO solution in the presence of minimal amount of water ( $\mathrm{ca}$. $2 \mathrm{wt} \%$ ) to dissolve a catalyst, sodium hydroxide, in the medium, and with the addition of an excess amount of vinyl sulfoxides or vinyl sulfones compared to the hydroxyl group concentration of poly(vinyl alcohol). The almost complete substitution of hydroxyl groups on poly(vinyl alcohol) was thus attained with 8 times excess of methyl or ethyl vinyl sulfoxides, which 3 times excess was sufficient with methyl and ethyl vinyl sulfones.

A series of sulfoxide- and sulfone-modified poly(vinyl alcohol) derivatives with various degrees of substitution was then subjected to ${ }^{13} \mathrm{C}$ NMR measurements. The methine region spectra of vinyl alcohol units in the reaction products between either methyl vinyl sulfoxide or methyl vinyl sulfone and poly(vinyl alcohol) were shown in Figure 1. The methine carbon signals in vinyl alcohol units were observed to 
a

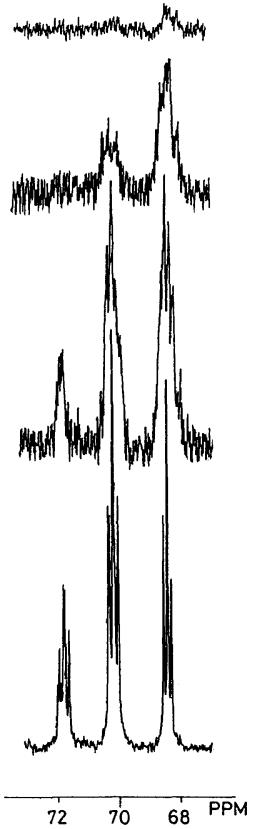

b

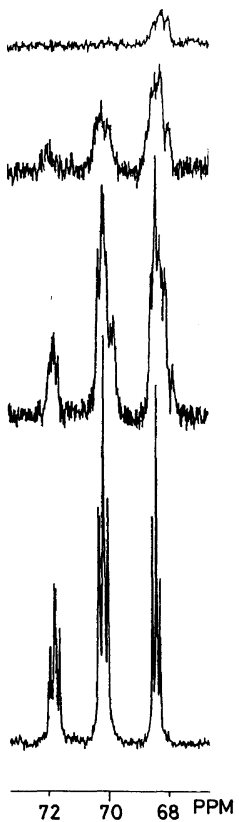

Figure 1. ${ }^{13} \mathrm{C}$ NMR methine region spectra of vinyl alcohol units in the reaction products of poly(vinyl alcohol) with (a) methyl vinyl sulfoxide and with (b) methyl vinyl sulfone at various degrees of substitution. (The degree of substitution for (a): $83,55,32$, and $0 \%$, and for (b): 81 , 46,26 , and $0 \%$ from the top to the bottom, respectively.)

resolve into three major peaks for the whole examined range of the degree of substitution and were scarcely affected by the introduction of sulfinyl- or sulfonylethyl substituted monomer units. Thus the methine carbon signals on vinyl alcohol units were remained to be indicative to their tactic environment, i.e., at 71.8 (isotactic), 70.2 (heterotactic), and 68.4 (syndiotactic) ppm, respectively. ${ }^{17}$ On the other hand, the methine carbon signals on sulfinyl- and sulfonylethyl derivative units appeared as complicated multiplets at around $78 \mathrm{ppm}$, presumably due to the influence of the comonomer distribution pattern and the tactic arrangements. One could therefore estimate the relative content of hydroxyl groups in each tactic sequence at various degrees of substitu- tion from the analysis of the methine carbon signals on vinyl alcohol units. The results for the reaction products of poly(vinyl alsohol) with two different types of vinyl sulfoxides and vinyl sulfones having methyl and ethyl substituents were summarized in Figure 2. It is clearly seen that hydroxyl groups located at the isotactic sequence were preferentially consumed at the early stage of the reaction in all examples, while those at the heterotactic sequence and in particular those at the syndiotactic sequence were apparently less reactive.

In Figure 3, the relative content of hydroxyl groups in each tactic sequence at various degrees of substitution was listed for a series of sulfoxide-modified poly(vinyl alcohol)s obtained by the reaction with methyl, ethyl, $t$-butyl and phenyl vinyl sulfoxides, respectively. This indicates that the reactivity of hydroxyl groups at the sequential unsubstituted vinyl alcohol units is not appreciably influenced by the bulkiness of the substituent in vinyl sulfoxides. Thus the kinetic retardation observed particularly in the reaction with $t$-butyl vinyl sulfoxide is thought to be caused by the steric hinderance of the introduced $t$-butyl groups on polymer chain to suppress the further access of the vinyl sulfoxide toward the neighboring hydroxyl groups. Besides, the introduction of alkylsulfinyl ethyl groups on poly(vinyl alcohol) causes the polymer coil shrinkage $^{1}$ and this may reduce the reactivity of hydroxyl groups at the late stage of the reaction.

The observed higher reactivity of hydroxyl groups at the isotactic sequence on poly(vinyl alcohol) may be accounted by the conformational consideration of isotactic and syndiotactic sequences in poly(vinyl alcohol), ${ }^{18}$ where the isotactic $(\mathrm{mm})$ sequence prefers the TG+GT conformation, while the TTTT conformation prevails in the syndiotactic $(r r)$ sequence in polar solvents like DMSO and $\mathrm{H}_{2} \mathrm{O}$. Consequently, hydroxyl groups at the isotactic sequence are considered to be less 
a

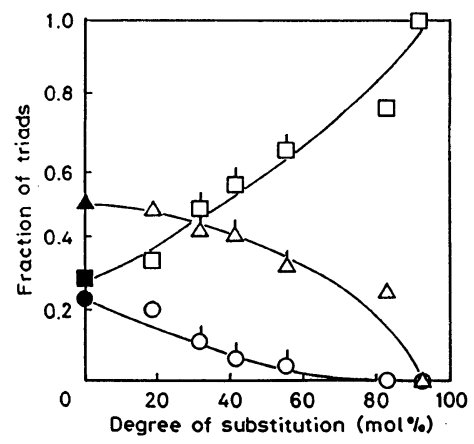

b

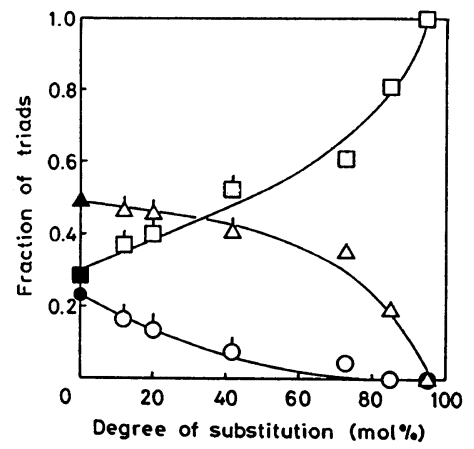

C

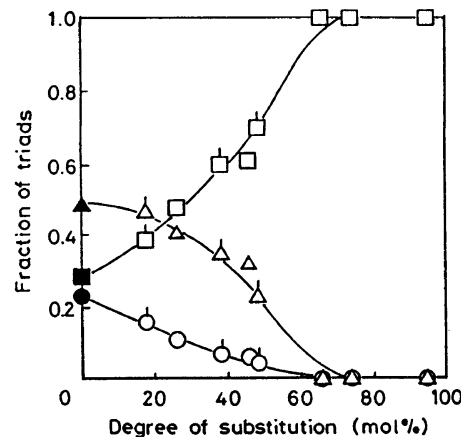

d

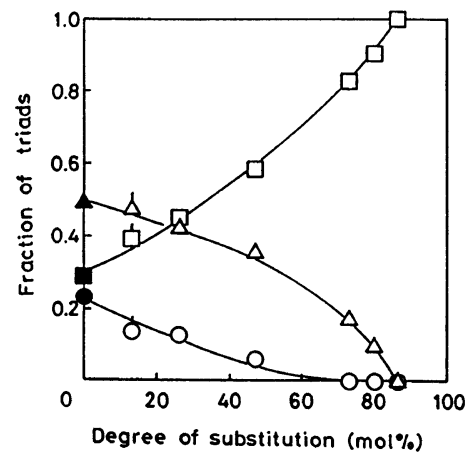

Figure 2. The fraction of tactic triads in poly(vinyl alcohol) and in the reaction products of poly(vinyl alcohol) with (a) methyl vinyl sulfoxide, (b) ethyl vinyl sulfoxide, (c) methyl vinyl sulfone, and (d) ethyl vinyl sulfone at various degrees of substitution. $(\boldsymbol{O}, \bigcirc, \bigcirc)$, isotactic; $(\boldsymbol{\Delta}, \triangle, \triangle)$, heterotactic; $(\square, \square$, $\square)$, syndiotactic fractions. Samples were prepared either in water at $60^{\circ} \mathrm{C}(\mathrm{O}, \triangle, \square)$ or in DMSO at $25^{\circ} \mathrm{C}(\bigcirc, \triangle, \square)$.
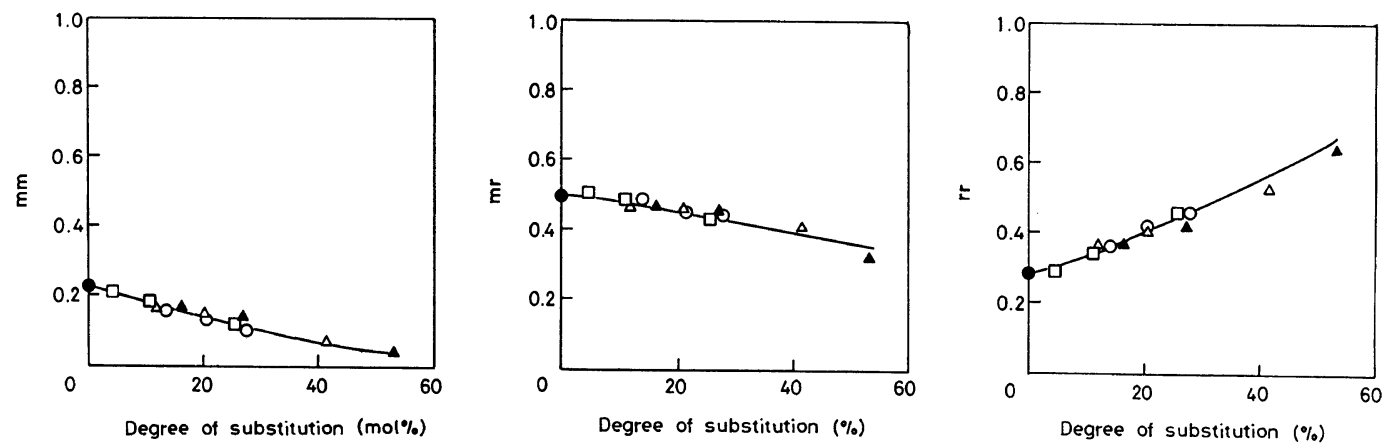

Figure 3. The fraction of tactic trisds in (O) poly(vinyl alcohol) and in the reaction products with $(O)$ methyl, $(\triangle)$ ethyl, $(\square) t$-butyl, and $(\boldsymbol{\Delta})$ phenyl vinyl sulfoxides. 


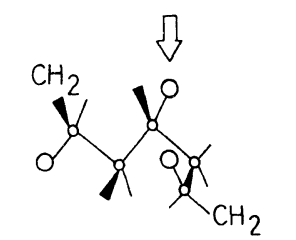

$\mathrm{mm}$ ( TGTG or GTGT)

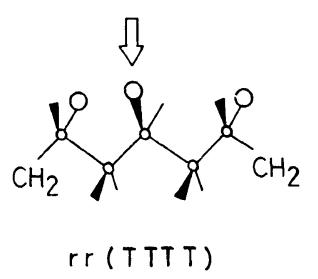

Figure 4. The chain conformation of the isotactic $(\mathrm{mm})$ and syndiotactic $(r r)$ sequences in poly(vinyl alcohol). (O) denotes the hydroxyl group.

sterically hindered internally than those at the syndiotactic counterpart as schematically shown in Figure 4. And hence, the former can react more rapidly than the latter in the addition reaction with vinyl sulfoxides and with vinyl sulfones.

Acknowledgements. A financial support from the Iwatani Naoji Foundation is gratefully acknowledged.

\section{REFERENCES}

1. K. Imai, T. Shiomi, Y. Tezuka, K. Takahashi, and M. Satoh, J. Macromol. Sci.-Chem., A22, 1359 (1985).

2. K. Imai, T. Shiomi, Y. Tezuka, and M. Takada, J.
Appl. Polym. Sci., 35, 1817 (1977).

3. K. Imai, T. Shiomi, Y. Tezuka, and M. Satoh, Makromol. Chem., Rapid Commun., 6, 413 (1985).

4. K. Imai, Y. Tezuka, T. Shiomi, and M. Takada, Bull. Chem. Soc. Jpn., 60, 653 (1987).

5. K. Imai, T. Shiomi, Y. Tezuka, and K. Inokami, Polymer International, in press.

6. K. Imai, T. Shiomi, Y. Tezuka, and K. Inokami, Sen-i Gakkaishi, in press.

7. J. Millan, G. Martinez, and C. Mijangos, J. Polym. Sci., Polym. Chem. Ed., 23, 1077 (1985).

8. G. Martinez, C. Mijangos, and J. L. Millan, Makromol. Chem., Rapid Commun., 7, 15 (1986).

9. G. Martinez, C. Mijangos, and J. Millan, Polym. Bull., 13, 151 (1985).

10. F. A. Jameison, F. C. Schilling, and A. E. Tonelli, Macromolecules, 19, 2168 (1986).

11. S. Boiven, P. Hemery, and S. Boileau, Am. Chem. Soc., Polym. Prepr., 27(2), 3 (1986).

12. I. Sakurada, Y. Sakaguchi, and Z. Shiiki, Kobunshi Kagaku, 21, 289 (1964).

13. K. Shibatani and K. Fujii, J. Polym. Sci., A-1, 8, 1647 (1970).

14. K. Fujii, J. Ukida, and M. Matsumoto, J. Polym. Sci., B., 1, 693 (1963); Maḱromol. Chem., 65, 86 (1963).

15. D. Vofsi, S. Yanai, and I. Cabasso, J. Polym. Sci., Polym. Chem. Ed., 22, 3335 (1984).

16. N. Kanamura, J. Appl. Polym. Sci., 33, 2065 (1987).

17. T. K. Wu and M. L. Sheer, Macromolecules, 10, 529 (1977).

18. T. Moritani and Y. Fujiwara, J. Chem. Phys., 59, 1175 (1973). 